\title{
Numerical Simulation of Bone Remodelling in the Human Mandible Surrounding of a Dental Implant
}

\author{
Zhiqiang Lian \\ State Key Laboratory of Structural Analysis \\ for Industrial Equipment \\ Dalian University of Technology \\ Dalian, China
}

\author{
Hong Guan \\ Griffith School of Engineering \\ Griffith University Gold Coast Campus \\ Queensland, Australia
}

\begin{abstract}
Dental implants provide the most efficient and economic long-term solution for replacing lost teeth. However, placement of an implant changes the normal mechanical environment of jawbone, which causes the bone density to redistribute and adapt to the new environment by remodelling. This study aims to predict the density distribution in human jawbone surrounding a dental implant. Based on the two popular, yet distinctive theories for bone remodelling, a new remodelling algorithm is proposed The proposed algorithm is verified by a two-dimensional (2D) plate model. Then, a 2D finite element model of implant and jawbone is studied. The effects of two parameters, viz the reference value of strain energy density (SED) and 'lazy zone' region, on the density distribution are also investigated. This study has demonstrated that consideration of the lazy zone, is less important than consideration of the stress and strain (quantified as SED) induced within the bone. The proposed bone remodelling algorithm is a combination and further development of the two popular but distinctive bone remodelling theories. The shortcomings of the two theories have been overcome in the proposed algorithm.
\end{abstract}

Keywords-bone remodelling; density distribution; implant-bone system; finite element technique

\section{INTRODUCTION}

Implant bone restorations have become a clinically and scientifically accepted treatment modality over the past 30 years. The realization that implants made of commercially pure titanium attain direct bone to implant contact initiated this revolution in oral rehabilitation.

Bone, as a living tissue, undergoes continuous adaptation and self-repair by resorption and formation, is capable of optimizing its internal structure under the influence of external loads. The self-adaptation process, termed as 'remodelling', has an enormous effect on the overall behavior of the entire bone tissue throughout an individual's lifetime. Following an early publication of Wolff [1], many theories describing the bone-adaptation process have been proposed. Carter suggested that bone is 'lazy' in terms of poor reaction to mechanical signals [2], and there was recognition of the existence of a 'lazy zone' representing the range of stimulus within which no remodelling occurs. The concept of 'lazy zone', recognising that a threshold stimulus needed to be exceeded for bone adaptation to occur, was incorporated by Huiskes et al. [3] who used the term strain energy density (SED) to describe the stresses and strains that control bone remodelling through bone formation and resorption. This theory has been developed further by Weinans et al. [4] and Turner et al. [5]. Mullender et al. [6] and Mullender and Huiskes [7] suggested a physiological approach to simulate bone remodelling which assumed that osteocytes are sensitive to mechanical loading and are active in controlling bone adaptation. While these theories were essentially developed for orthopedics, they are generally applicable to jawbone surrounding a dental implant [8]. However, work in this area is limited. Mellal et al.[9] used three different stimuli (equivalent strain, equivalent stress and SED) to predict bone remodelling around a dental implant based on the existing theories and the results were compared with in vivo data. Li et al.[10] developed a new bone remodelling algorithm by introducing an additional quadratic term based on the theory of Weinans et al.[4], which can simulate both underload and overload resorption. The algorithm was applied in conjunction with the FEM to a practical case of dental implant treatment. When the implant is placed into mandible, the mechanical environment of jawbone changes accordingly, hence the balance of bone resorption and formation is no longer maintained. From a clinical viewpoint, better understanding of the way that functional forces can affect the remodelling of bone is important in terms of identifying minimal and maximal loading forces required for the maintenance of stable osseointegration during function, as well as preventing overload that can lead to implant failure.

In this paper, a new algorithm for bone remodelling is proposed based on the abovementioned theories. The proposed algorithm is verified by a 2D plate model simulating bone tissue studied previously by Weinans et al.[4] and Mullender et al.[6], which confirms its accuracy and reliability. To demonstrate the ability of the proposed algorithm in predicting the density distribution of bone surrounding a dental implant, a 2D finite element model of implant and jawbone is studied. The effects of two parameters viz the reference value of SED and 'lazy zone' region, on the density distribution are also investigated and discussed in some detail. 


\section{MATERIALS AND METHODS}

\section{A. The Existing Bone Remodelling Theory}

Among a number of bone remodelling theories[3-7,11-13], two popular yet distinctive ones are summarized in the following subsections followed by the presentation of the proposed algorithm.

1) Huiskes and Weinans Theory: A popular bone remodelling theory suggested by Huiskes et al.[3] and Weinans et al. [4] states that:

$$
\frac{d \rho}{d t}=\left\{\begin{array}{ccc}
B\left(\frac{U}{\rho}-(1+s) K\right) & \text { if } & \frac{U}{\rho}>(1+s) K \\
0 & \text { if } & (1-s) K \leq \frac{U}{\rho} \leq(1+s) K \\
B\left(\frac{U}{\rho}-(1-s) K\right) & \text { if } & \frac{U}{\rho}<(1-s) K
\end{array}\right.
$$

where $d \rho / d t$ is the rate of change in density; $\rho$ is the apparent density of bone tissue; $B$ is a constant regulating the rate of the remodelling process; $s$ is in percentage denoting the region of the 'lazy zone' around the threshold value $K$, which is a reference value for SED.

This algorithm was applied to predict the density distribution in the normal proximal femur with results showing a discontinuous pattern [4], a phenomenon called 'checker-board'.

2) Mullender et al's Theory: Not having intrinsically different biological nature from the theory of Huiskes et al.[3] and Weinans et al.[4], Mullender et al.[6,7] suggested a new algorithm which simulated bone remodelling as a self-organisational control process. In their theory, the bone is assumed to have $N$ sensor cells distributed uniformly over its volume. An arbitrary sensor $i$ measures a signal $S_{i}$, which is given as:

$$
S_{i}=\frac{U_{i}}{\rho_{i}}
$$

The density $\rho(\mathbf{x}, t)$ at location $\mathbf{x}$ is regulated by the stimulus value $\Phi(\mathbf{x}, t)$, to which all sensor cells contribute, relative to their distance from $\mathbf{x}$. Hence,

$$
\Phi(\mathbf{x}, t)=\sum_{i=1}^{N} f_{i}(\mathbf{x})\left(S_{i}-K\right)
$$

where $N$ is the total number of sensors; $K$ is a reference signal of SED and $f_{i}(\mathbf{x})$ is a spatial influence function given as:

$$
f_{i}(\mathbf{x})=e^{-\left[d_{i}(\mathbf{x}) / D\right]}
$$

where $d_{i}(\mathbf{x})$ is the distance between sensor $i$ and location $\mathbf{x}, D$ is the rate of the spatial influence reduction.

The density $\rho(x, t)$ is now governed by the rate:

$$
\frac{d \rho(\mathbf{x}, t)}{d t}=\tau \Phi(\mathbf{x}, t), \text { with } 0<\rho(x) \leq \rho_{\max }
$$

where $\rho_{\max }$ is the maximum density of cortical bone, and $\tau$ is a time constant regulating the rate of the process.

This algorithm was used to predict the density distribution for a $2 \mathrm{D}$ plate model previously studied by
Weinans et al.[4]. The model produced trabecular-like structures without the 'checker-board' patterns.

\section{B The Proposed Algorithm}

The existing two theories have both advantages and disadvantages. Huiskes and Weinans's theory takes into account the 'lazy zone' effect but it is unstable in its mathematical operation leading to a discontinuous and an inevitable 'checker board' pattern. Mullender's theory, on the other hand, is physiologically and mechanically more consistent and the 'checker board' phenomena can be effectively eliminated. However the effect of 'lazy zone' was not considered. The 'lazy zone' effect, initially proposed by Carter[2] based on experimental investigation, has been verified by Rubin and Lanyon [14] to be an important factor.

To overcome the shortcomings of the above two theories, a new algorithm is proposed herein. The following governing equation is proposed, taking into account the 'lazy zone' effect. For an arbitrary sensor $i$,

$$
\frac{d \rho_{i}(\mathbf{x}, t)}{d t}=\tau \Phi_{i}(\mathbf{x}, t)
$$

and

$$
\Phi_{i}(\mathbf{x}, t)=\left\{\begin{array}{ccc}
\sum_{i=1}^{N} f_{i}(\mathbf{x})\left(\frac{U_{i}}{\rho_{i}}-(1+s) K\right) & \text { if } & \frac{U_{i}}{\rho_{i}}>(1+s) K \\
0 & \text { if } & (1-s) K \leq \frac{U_{i}}{\rho_{i}} \leq(1+s) K \\
\text { with } 0<\rho_{i}(x) \leq \rho_{\max } & \frac{U_{i}}{\rho_{i}}<(1-s) K
\end{array},\right.
$$

where $f_{i}(\mathbf{x})$ is the spatial influence function in the same form as in (4).

As the apparent density of bone tissue changes during the process of remodelling, the Young's modulus changes accordingly. Some empirical relationships between the apparent density and the mechanical properties of bone have been established experimentally $[15,16]$. The Young's modulus $E$ at location $\mathbf{x}$ is calculated according to Carter and Hayes' study [15] as:

$$
E(x, t)=3790 \times \rho^{3}
$$

In the iterative finite element analysis process, the governing equation (6) is necessary to be rewritten as:

$$
\rho_{i}(t+\Delta t)=\rho_{i}(t)+\Delta t \tau \Phi_{i}(t)
$$

where $\Delta t$ is the time step in the iteration process; $\Phi(t)$ is in the same form as in (7). The iteration is continued until no more significant changes occur in the density distribution.

\section{RESULTS}

A 2D representation of the implant and mandible is studied herein and the modelling scheme follows that of a previous study [17].

The different types of bone, i.e. cortical and cancellous bone are identified in the model based on CT images. The implant dimensions are based on those of Neoss implant (Neoss Limited, UK). Fig. 1a shows the 
dimensions of the entire implant and jawbone model. The implant is conical with 2 degrees of taperage and has a helical thread. The load and boundary conditions shown in Fig. 1b are based on the work of Chou et al.[18] in which the implant system is loaded with an occlusal load of $100 \mathrm{~N}$ on the crown at an angle of $11^{\circ}$ and a uniformly distributed pressure of $500 \mathrm{kPa}$ on the outer surface of the cortical bone.

Typical values of Young's modulus $(E)$ of bone and implant for the analysis are selected based on the work of Papavasiliou et al.[19], which are also detailed in Fig. 1b. The Poisson's ratio for all components is taken as 0.3 . Note that in this study a perfect connection between the implant and jawbone is assumed.

The remodelling is considered for cancellous bone only with a uniform initial density distribution of $\rho=1.4$ $\mathrm{g} / \mathrm{cm}^{3}$ which corresponds to $E=7.93 \mathrm{GPa}$.

The maximum and minimum densities are $\rho_{\text {max }}=1.5347 \mathrm{~g} / \mathrm{cm}^{3}$ and $\rho_{\text {min }}=0.064 \mathrm{~g} / \mathrm{cm} 3$ respectively which corresponds to Young's modulus of $13.7 \mathrm{GPa}$ and $1 \mathrm{kPa}$. The 'lazy zone' effect $s$ in (7) is taken as 0.1 , the constant $\tau=1\left(\mathrm{~g} / \mathrm{cm}^{3}\right)^{2} /(\mathrm{MPa}$ time-unit). The influence parameter $D=0.25 \mathrm{~mm}$.

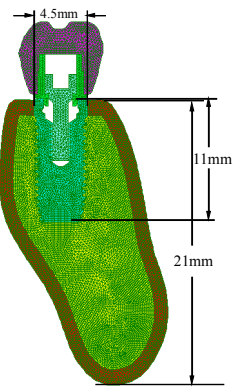

a)

Figure 1. Finite element model: a) dimensions; b) load, boundary conditions and material properties

To evaluate the effect of 'lazy zone' on the density distribution, different values of $s$ are considered with a constant SED value $K=0.0052 \mathrm{~J} / \mathrm{g}$. The predicted results are shown in Fig. 2. When parameter $s$ increases, the region of 'lazy zone' expands accordingly - hence more bone tissue remains 'lazy', i.e. neither resorption nor formation. This corresponds to a larger grey region on the lingual side of the implant (Fig. 2d). Although more 'lazy zone' is evident when $s$ increases, the overall morphology does not vary significantly.

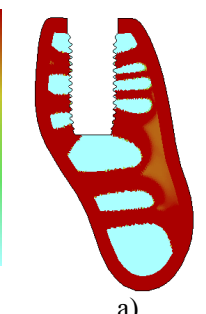

a)

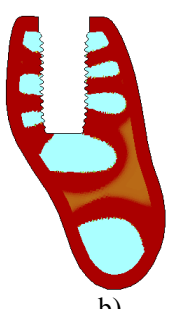

b)

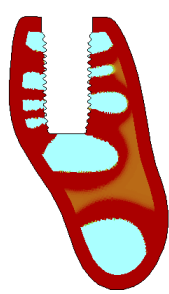

c)

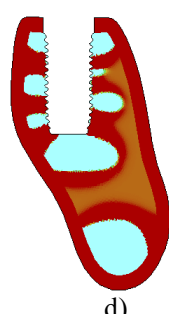

d)
Figure 2. Influence of 'lazy zone' effect on the density distribution: a) $\mathrm{s}=0.1$; b) $\mathrm{s}=0.2$; c) $\mathrm{s}=0.3$; d) $\mathrm{s}=0.4$

Different reference values $K$ in (7) are also considered to evaluate the effects of threshold value of
SED on the density distribution. The results shown in Fig. 3 indicate that when $K$ increases, more bone tissue is resorbed in the surrounding jawbone, especially on the lingual side of the implant due to the oblique loading condition.

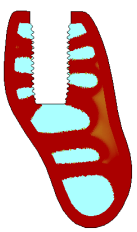

a)

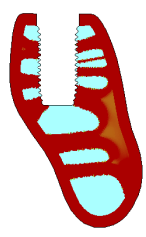

b)

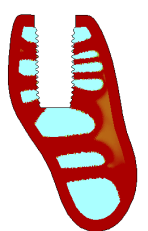

c)

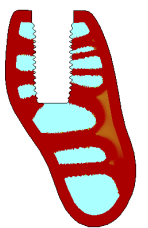

d)

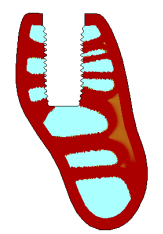

e)
Figure 3. Influence of reference SED value $\mathrm{K}$ on the density distribution of jawbone surrounding dental implant: a) $\mathrm{K}=0.0048$ $\mathrm{J} / \mathrm{g}$; b) $\mathrm{K}=0.005 \mathrm{~J} / \mathrm{g}$; c) $\mathrm{K}=0.0052 \mathrm{~J} / \mathrm{g}$; d) $\mathrm{K}=0.0054 \mathrm{~J} / \mathrm{g}$; e) $\mathrm{K}=0.0056 \mathrm{~J} / \mathrm{g}$

Fig. 4 shows the relationship between the bone mass (for cancellous bone only) and the two parameters $s$ and $K$. When $s$ increase by $300 \%$, the weight $w$ only increase $2.08 \%$, correspondingly, when $k$ increase by $16.7 \%$, the weight $w$ decrease $4.5 \%$. It can be deduced that the bone mass increases slightly with an increase in the 'lazy zone' effect $s$ (Fig. 4a) and decreases more noticeably with an increase in the reference value $K$ (Fig. 4b). It can be deduced from Fig. 4 that the parameter $K$ has more significant effect than $s$ on bone remodelling.

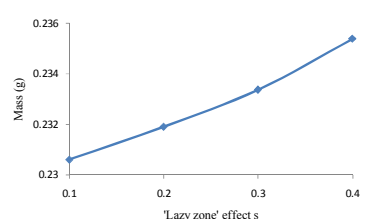

a)

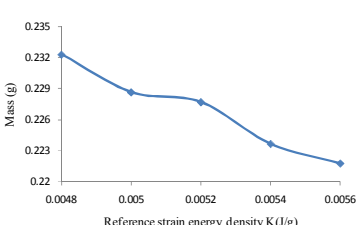

b)
Figure 4. Influence of two parameters on bone mass: a) 'lazy zone' effect $s ;$ b) reference value $K$

\section{DISCUSSION}

This paper proposes a new algorithm for the study of bone remodelling, which is a combination and further development of two existing and widely accepted bone remodelling theories. The algorithm takes into account both the 'lazy zone' effect and the self-organisational control process. It is shown to be accurate and reliable upon verification with a plate model simulating bone tissue. The algorithm is then used to predict the density distribution in human jawbone surrounding a dental implant. The influence of two parameters viz the reference SED value $K$ and the 'lazy zone' effect $s$ on the density distribution is also evaluated. The results indicate that $K$ has more significant influence than $s$.

With an increasing value of $K$, a reduction in bone formation and an increase in bone resorption are evident, and vice versa. There is no general rule or criterion in published literature on the selection of the reference value $K$. It is found in this study that a median value among all the SEDs is appropriate to yield a satisfactory density distribution outcome.

Fig. 5 compares the predicted density distribution in cancellous bone surrounding a dental implant with a published clinical observation by Watzak et al.[20] and a 
numerical prediction by Chou et al.[18]. Fig. 5a shows Watzak's clinical observation of density distribution on baboons and Fig. 5b presents Chou et al.'s bone remodelling prediction around a dental implant. The predicted result (with $K=0.0052 \mathrm{~J} / \mathrm{g}$ ) due to the proposed algorithm is re-produced in Fig. 5c. The overall density distributions are quite similar for three cases in terms of the trabecular-like pattern and the blank area below the implant. This is particularly true when comparing Fig. 5b and Fig. 5c. However, due to the simplification of the finite element model, discrepancies in density distribution do exist between the present computational modelling outcome and the clinical observation.
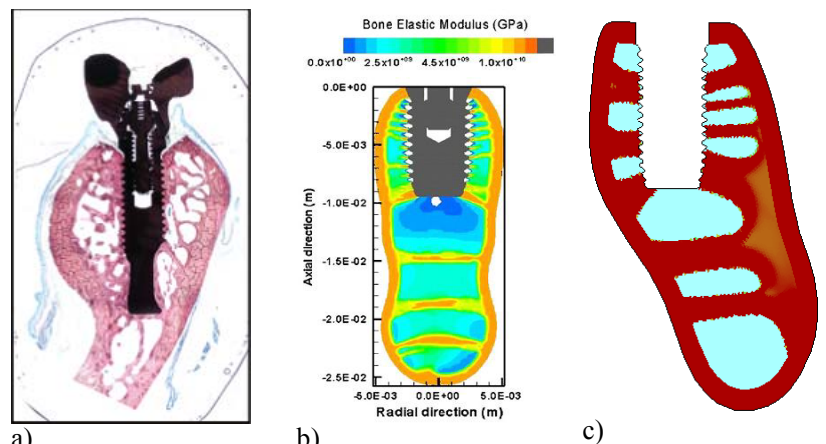

b)

c)

Figure 5. Comparison of predicted result with clinical observations. a) density distribution on baboons; b) Chou et al.'s[31] prediction; c) predicted result with $\mathrm{K}=0.0052 \mathrm{~J} / \mathrm{g}$

It should be noted that this study is a preliminary attempt in developing a new bone remodelling algorithm suitable for computer simulation of the highly complex processes of bone healing and remodelling. In addition to the assumed perfect implant/bone connection, this study also employs a simplified and fixed loading condition. This is considerably different from the actual masticatory forces which always vary in both magnitude and direction. Hence, a discrepancy between the resulting density distribution (Fig. 5) and the true anatomy of jawbone is expected. Further work should be done to evaluate the effects of other parameters which may also affect the density distribution within the jawbone surrounding a dental implant.

\section{CONCLUSIONS}

Whilst further optimization of the algorithm described here is desirable, our model has been shown to correlate well with clinical observations. An important consideration is that the 'lazy zone' is less important than consideration of the 'strain energy density'. Improved understanding of the factors which influence bone remodelling around osseointegrated dental implants is important in quantifying the forces compatible with maintaining successful osseointegration and preventing overload leading to implant failure. To this end, the newly developed algorithm incorporating elements from previous theories more closely replicates the clinical situation.

\section{ACKNOWLEDGEMENTS}

The financial support from the 2006 Griffith University Research Grant scheme and the assistance of Griffith PhD scholar Mr. Rudi van Staden are gratefully acknowledged.

\section{REFERENCES}

[1] J. L. Wolff, The law of bone remodelling. Translated by Maquet P, Furlong R, in 1986. Berlin: Springer-Verlag, 1892.

[2] D. R. Carter, "Mechanical loading histories and cortical bone remodelling," Calcif. Tissue Int., Vol. 36, pp. 19-24, 1984.

[3] R. Huiskes, H. Weinans, H. J. Grootenboer, M. Dalstra, B. Fudala, and T. J. Slooff, "Adaptive bone-remodeling theory applied to prosthetic design analysis," J. Biomech., Vol. 20, pp. 1135-1150, 1987.

[4] H. Weinans, R. Huiskes, and H. J. Grootenboer, "The behavior of adaptive bone-remodeling simulation models," J. Biomech., Vol. 25, pp. 1425-1441, 1992.

[5] C. H. Turner, V. Anne, and R. M. V. Pidaparti, "A uniform strain criterion for trabecular bone adaptation, do continuum-level strain gradients drive adaptation," J. Biomech., Vol. 30, pp. 555-563, 1997.

[6] M. G. Mullender, R. Huiskes, and H. Weinans, "A physiological approach to simulation of bone remodelling as a self-organizational control process," J. Biomech., Vol. 27, pp. 1389-1394, 1994.

[7] M. G. Mullender, and R. Huiskes, "Proposal for the regulatory mechanism of Wolff's law,” J. Orthop. Res., Vol. 13, pp. 503-512, 1995.

[8] J. Duyck, H. J. Ronold, H. Van Oosterwyck, I. Naert, J. V. Sloten, and J. E. Ellingsen, "The influence of static and dynamic loading on marginal bone reactions around osseointegrated implants: an animal experimental study," Clin. Oral Implants Res., Vol. 12, pp. 207-218, 2001.

[9] A. Mellal, H. W. A. Wiskott, S. S. Scherrer, and U. C. Belser, "Stimulating effect of implant loading on surrounding bone. Comparison of three numerical models and validation by in vivo data," Clin. Oral. Implants Res., Vol. 15 pp. 239-248, 2004.

[10] J. Li, H. Li, L. Shi, A. S. L. Fok, C. Ucer, H. Devlin, et al., "A mathematical model for simulating the bone remodelling process under mechanical stimulus," Dent. Mater., Vol. 23, pp. 1073-1078, 2007.

[11] R. T. Hart, and D. T. Davy, Theories of bone modelling and remodelling. 1st ed.,S. C. Cowin, Bone Mechanics, Boca Raton: CRC Press; 1989.

[12] D. P. Fyhrie, and D. R. Carter, "Femoral head apparent density distribution predicted from bone stresses," J. Biomech, Vol. 23, pp. 1-10, 1990.

[13] C. R. Jacobs, J. C. Simo, G. S. Bearpre, and D. R. Carter, "Adaptive bone remodeling incorporating simultaneous density and anisotropy considerations," J. Biomech., Vol. 30, pp. 603-613, 1997.

[14] C. T. Rubin, and L. E. Lanyon, "Regulation of bone mass by mechanical strain magnitude," Calcif. Tissue Int., Vol. 37, pp. 411-417, 1985

[15] D. R. Carter, and W. C. Hayes, "The compressive behavior of bone as a two-phase porous structure," J. Bone Joint Surg., Vol. 59, pp. 954-962, 1977.

[16] E. F. Morgan, H. H. Bayraktar, and T. M. Keaveny, "Trabecular bone modulus-density relationships depend on anatomic site," J. Biomech., Vol. 36, pp. 897-904, 2003.

[17] H. Guan, R. van Staden, Y. C. Loo, N. W. Johnson, S. Ivanovski, and N. Meredith, "Influence of bone and dental implant parameters on stress distribution in mandible - A finite element study," Int. J. Oral Maxillofac. Implants, Vol. 24, pp. 866-876, 2009.

[18] H. Y. Chou, J. J. Jagodnik, and S. Muftu, "Predictions of bone remodelling around dental implant systems," J. Biomech., Vol. 41, pp. 1365-1373, 2008.

[19] G. Papavasiliou, P. Kamposiora, S. C. Bayne, and D. A. Felton, "3D-FEA of osseointegration percentages and patterns on implant-bone interfacial stresses,” J. Dent., Vol. 25, pp. 485-491, 1997.

[20] G. Watzak, W. Zechner, C. Ulm, S. Tangl, G. Tepper, and G. Watzek, "Histologic and histomorphometric analysis of three types of dental implants following 18 months of occlusal loading: a prelimary study in baboons," Clin. Oral Implants Res., Vol. 16, pp. 408-416, 2005 